\title{
Stability Theory for Solitary-Wave Solutions of Scalar Field Equations
}

\author{
Daniel B. Henry ${ }^{1 \star}$, J. Fernando Perez ${ }^{2 \star \star}$, and Walter F. Wreszinski ${ }^{2 \star \star}$ \\ 1 Instituto de Matemática, Universidade de São Paulo, São Paulo, Brazil \\ 2 Instituto de Física, Universidade de São Paulo, São Paulo, Brazil
}

\begin{abstract}
We prove stability and instability theorems for solitary-wave solutions of classical scalar field equations.
\end{abstract}

\section{Introduction}

In this paper, we study the stability of special travelling-wave ("solitary-wave", [1]) solutions of classical scalar field equations of the form

$$
\square \phi+U^{\prime}(\phi)=0 .
$$

This problem has attracted much attention recently in the physics literature ([2]), in part because classical solutions may be recovered from suitable expectation values of quantum fields in the classical limit ([3]).

Apart from the main motivation, which is to provide a simple and clear mathematical theory of stability for classical field equations, there is also a three-fold physical motivation. Firstly, most of the discussion in the physics literature ([2]), which is heuristically correct, relies on the linear theory. It may be shown, however, using methods of the present paper, that the latter is not applicable, because the linearized operator (on the natural Hilbert space, after proper "subtraction" of the zero mode) is skew-adjoint, a reflection of the fact that the mechanism of stability in these theories is dispersive, not dissipative (see also the discussion in [6] for K-dV equation). Secondly, the existing rigorous nonlinear stability theories ([4], generalized and corrected in [5], and [6]) are in principle applicable only to a class of equations (such as the K-dV equation) which may be treated either by inverse scattering theory $([6])$, or which possess more than one scalar conservation law ([4], [5]), and are, therefore, unsuitable to describe, for instance, the stability of "kinks" of the nonlinear Klein-Gordon equation ([2]). Thirdly, and perhaps most importantly, the heuristic discussion disregards the somewhat delicate technical problems posed

* Partial financial support by FAPESP and FINEP.

$\star \star$ Partial financial support by CNPq. 
by the zero-mode, which is always present due to translation invariance ([2]): this is also the reason why stability of the solitary-waves is a form-stability ([4], [6]).

The plan of the paper is as follows. In Sect. 1 we describe the relevant class of equations and solutions, state and prove our stability result (Theorem 1), and in Sect. 2 we prove a general instability result (Theorem 2). In Sect. 3 we provide a brief discussion of applications of Theorems 1 and 2 and prove, in particular, instability of the solitary-waves in higher dimensions which have been constructed by Parenti et al. ([13]) and Strauss ([17]). The Derrick-Strauss theorem ([7],[8]) is thereby revisited.

\section{Stability}

Let $\phi$ be a bounded static (time-independent) solution of (0-1), i.e., satisfying

$$
\phi^{\prime \prime}(x)=U^{\prime}(\phi(x)), \quad x \in \mathbb{R} .
$$

We further assume

$$
\phi^{\prime}(x)>0, \quad \forall x \in \mathbb{R}, \quad \phi^{\prime}(x) \underset{x \rightarrow \pm \infty}{\rightarrow} 0,
$$

and that there exist constants $-\infty<a_{-}<a_{+}<\infty$ such that

$$
\phi(x) \underset{x \rightarrow-\infty}{\rightarrow} a_{-} \text {and } \phi(x) \underset{x \rightarrow+\infty}{\rightarrow} a_{+} .
$$

Since $U(\phi(x))=\frac{1}{2} \phi^{\prime}(x)^{2}+$ const, it follows that $U\left(a_{-}\right)=U\left(a_{+}\right)$. We normalize the energy of the "vacua" $\phi(x) \equiv a_{+}$and $\phi(x) \equiv a_{-}$to zero and assume

$$
U\left(a_{-}\right)=U\left(a_{+}\right)=0 \text { and } U(x) \geqq 0, \quad x \in \mathbb{R} .
$$

We also assume that

$$
E_{0} \equiv \int_{-\infty}^{\infty}\left(\frac{1}{2} \phi^{\prime 2}+U(\phi)\right)<\infty
$$

This means that $\phi$ has finite energy relative to the vacua. This point and the restriction to one space dimension are related to the Derrick-Strauss theorem ([7], [8]), see the discussion in Sect. 3. On $U$ we further impose the following condition:

$$
U \in C^{2} \text { in a neighbourhood of }\left[a_{-}, a_{+}\right] \text {and } U^{\prime \prime}\left(a_{ \pm}\right)>0 .
$$

We note that by the first of (1-2) we are describing "lumps" ([2]). "Antilumps" $\left(\phi^{\prime}(x)<0, x \in \mathbb{R}\right)$ may be handled by reflection $x \rightarrow-x$.

Let $H_{\mathrm{loc}}^{1}(\mathbb{R}) \equiv\left\{\psi: \mathbb{R} \rightarrow \mathbb{R}: \int_{\Omega} d x\left[\psi^{\prime}(x)^{2}+\psi(x)^{2}\right]<\infty\right.$ for every bounded region

$$
\begin{gathered}
\Omega \in \mathbb{R}\}, \\
H^{1}(\mathbb{R}) \equiv\left\{\psi: \mathbb{P} \rightarrow \mathbb{R}: \int_{-\infty}^{\infty} d x\left[\psi^{\prime}\left(x^{2}\right)^{2}+\psi(x)^{2}\right]<\infty\right\} .
\end{gathered}
$$


$H_{\text {loc }}^{1}(\mathbb{R})$ is the natural space for solutions of $(0-1)([12])$, and we shall therefore assume $\phi \in H_{\text {loc }}^{1}(\mathbb{R})$. Such a $\phi$ defines (under the previous assumptions) a Hilbert sector in the language of $([12])$, and it is natural to inquire upon stability within a sector, which means that "perturbed" solutions are required to satisfy $\left(\begin{array}{c}\psi-\phi \\ \psi_{t}\end{array}\right) \in H^{\prime}(\mathbb{R}) \oplus L_{2}(\mathbb{R})$. Due to translation invariance, the following is (for $q>0$ ) a natural distance function (see also [4]):

$$
d_{q}(\psi)^{2} \equiv \min _{-\infty<c<\infty} \int_{-\infty}^{\infty} d x\left[\left(\psi^{\prime}(x)-\phi^{\prime}(x+c)\right)^{2}+q(\psi(c)-\psi(x+c))^{2}\right]
$$

We may now state our stability theorem:

Theorem 1. There exist positive constants $r, q$ and $k$ such that if $u \in H_{\mathrm{loc}}^{1}(\mathbb{R})$ satisfies

$$
\left(u_{x}\left(., t_{0}\right), u_{t}\left(., t_{0}\right)\right) \in L_{2}(\mathbb{R}) \times L_{2}(\mathbb{R})
$$

and is a solution of (0-1) satisfying

$$
d_{q}\left(u\left(., t_{0}\right)\right)<r
$$

at some time $t_{0}$, having energy

$$
E=\int_{-\infty}^{\infty} d x\left[\frac{1}{2} u_{t}^{2}+\frac{1}{2} u_{x}^{2}+U(u)\right]<E_{0}+k r^{2},
$$

then the solution exists for all $t$ and has

$$
d_{q}(u(., t)) \leqq \sqrt{\frac{1}{k}\left(E-E_{0}\right)}<r
$$

for all $t$.

The theorem is a consequence of the following

Proposition 1. There exist positive constants $q, k$ and $r$ such that any solution $\psi \in H_{\mathrm{loc}}^{1}(\mathbb{R})$ of $(0-1)$ with $d_{q}\left(\psi\left(., t_{0}\right)\right) \leqq r$ satisfies

$$
\int_{-\infty}^{\infty} d x\left[\frac{1}{2} \psi^{\prime}(x)^{2}+U(\psi(x))\right] \geqq E_{0}+k d_{q}(\psi)^{2} .
$$

Proof. For clarity we divide the proof into a succession of steps:

1) $\phi^{\prime}(x)=0\left[e^{-\lambda_{ \pm}|x|}\right]$ as $x \rightarrow \pm \infty, \lambda_{ \pm}=\sqrt{U^{\prime \prime}\left(a_{ \pm}\right)}>0$, since $\left(\phi, \phi^{\prime}\right)=\left(a_{+}, 0\right)$ or $\left(a_{-}, 0\right)$ are saddle points of the equilibrium equation $(1-1)$.

2) The potential energy $V(\psi)=\int_{-\infty}^{\infty} d x\left(\frac{1}{2} \psi^{\prime 2}+U(\psi)\right)$ has $\phi$ as a critical point:

$$
\left(V^{\prime}(\phi), \psi\right)=\int_{-\infty}^{\infty} d x\left(\phi^{\prime} \psi^{\prime}+U^{\prime}(\phi) \psi\right)=0 \text { for all } \psi \in H^{1}(\mathbb{R})
$$

and

$$
\left(\psi, V^{\prime \prime}(\phi) \psi\right)=\int_{-\infty}^{\infty} d x\left(\psi^{\prime 2}+U^{\prime \prime}(\phi) \psi^{2}\right)=\int_{-\infty}^{\infty} d x\left(\psi A_{\phi} \psi\right)
$$


where $A_{\phi} \equiv-d^{2} / d x^{2}+U^{\prime \prime}(\phi(x))$ is a self-adjoint operator in $L_{2}(\mathbb{R})$, bounded below, with essential spectrum $\sigma_{e}\left(A_{\phi}\right)=[b, \infty], b=\min \left(U^{\prime \prime}\left(a_{+}\right), U^{\prime \prime}\left(a_{-}\right)\right)>0([9]$, Theorem 16, pg. 1448 and Theorem 4, pg. 1438). In particular, $0 \notin \sigma_{e}\left(A_{\phi}\right)$. If $\tau_{c}: \psi \rightarrow \psi(.+c)$ is the translation operator in $L_{2}(\mathbb{R})$ then $\tau_{c}^{-1} A_{\tau_{c} \phi} \tau_{c}=A_{\phi}$, so $\sigma\left(A_{\tau_{c} \phi}\right)=\sigma\left(A_{\phi}\right)$ for all $c$.

3) $\phi^{\prime \prime}=U^{\prime}(\phi)$ so $\phi^{\prime \prime \prime}=U^{\prime \prime}(\phi) \phi^{\prime}$, i.e. $A_{\phi} \phi^{\prime}=0$. By hypothesis $E_{0}<\infty$, hence $\phi^{\prime} \in L_{2}(\mathbb{R})$. Since $\phi^{\prime}>0,0$ is the smallest eigenvalue of $A_{\phi}$ and it is simple, by standard methods.

4) Let $\beta>0$ denote the first positive eigenvalue of $A_{\phi}$, or $b$, whichever is smaller. Then, for $\psi \in H^{1}(\mathbb{R}), \int_{-\infty}^{\infty} d x \psi \phi^{\prime}=0$ implies

$$
\left(\psi, V^{\prime \prime}(\phi) \psi\right)=\int_{-\infty}^{\infty} d x\left(\psi^{\prime 2}+U^{\prime \prime}(\phi) \psi^{2}\right) \geqq \beta \int_{-\infty}^{\infty} d x \psi^{2} .
$$

The same estimate holds (with the same $\beta$ ) when $\phi$ is replaced by $\Phi(.+c)$.

5) Suppose $q \geqq U^{\prime \prime}(\phi(x))$ for all $x$ and $\int_{-\infty}^{\infty} d x \psi \phi^{\prime}\left(q-U^{\prime \prime}(\phi)\right)=0$; then

$$
\left(\psi, V^{\prime \prime}(\phi) \psi\right)>\frac{\beta}{\left(1+K_{q}\right)^{2}} \int_{-\infty}^{\infty} d x \psi^{2}
$$

where

$$
K_{q} \equiv \frac{\left\|\phi^{\prime}\right\|_{L_{2}}\left\|\phi^{\prime}\left(q-U^{\prime \prime}(\phi)\right)\right\|_{L_{2}}}{\int_{-\infty}^{\infty} d x \phi^{\prime 2}\left(q-U^{\prime \prime}(\phi)\right)}
$$

To see this, set $\psi=\alpha \phi^{\prime}+\theta, \alpha=$ constant, $\int_{-\infty}^{\infty} d x \phi^{\prime} \theta=0$. Then

$$
\left(\psi, V^{\prime \prime}(\phi) \psi\right)=\left(\theta, V^{\prime \prime}(\phi) \theta\right) \geqq \beta \int_{-\infty}^{\infty} d x \theta^{2}
$$

and

$$
0=\alpha \int d x \phi^{\prime 2}\left(q-U^{\prime \prime}(\phi)\right)+\int d x \theta \phi^{\prime}\left(q-U^{\prime \prime}(\phi)\right)
$$

so

$$
\|\psi\|_{L_{2}} \leqq|\alpha|\left\|\phi^{\prime}\right\|_{L_{2}}+\|\theta\|_{L_{2}} \leqq\left(K_{q}+1\right)\|\theta\|_{L_{2}}
$$

6) $q \int_{-\infty}^{\infty} d x(u(x)-\phi(x+c))^{2} \rightarrow \infty$ as $c \rightarrow \pm \infty$, so there exists $c$ with $d_{q}(u)^{2}=$ $\int_{-\infty}^{\infty} d x\left[\left(u^{\prime}(x)-\phi^{\prime}(x+c)\right)^{2}+q(u(x)-\phi(x+c))^{2}\right]$. The minimum may be achieved at several values of $c$, but any one will serve, and we shall assume $c=0$ for simplicity. The derivative with respect to $c$ must vanish at $c=0$ and

$$
\begin{aligned}
0 & =\int_{-\infty}^{\infty} d x\left[\left(u^{\prime}-\phi^{\prime}\right) \phi^{\prime \prime}+q(u-\phi) \phi^{\prime}\right]=\int_{-\infty}^{\infty} d x(u-\phi)\left[q \phi^{\prime}-\phi^{\prime \prime}\right] \\
& =\int_{-\infty}^{\infty} d x(u-\phi) \phi^{\prime}\left(q-U^{\prime \prime}(\phi)\right),
\end{aligned}
$$


so with $\psi=u-\phi$ in step 5),

$$
\left((u-\phi), V^{\prime \prime}(\phi)(u-\phi)\right)=\left(\psi, V^{\prime \prime}(\phi) \psi\right) \geqq \frac{\beta}{\left(1+K_{q}\right)^{2}} \int_{-\infty}^{\infty} d x \psi^{2} .
$$

The same estimate holds with $\phi$ replaced by any translate $\phi(.+c)$.

7) Let $\psi \in H^{1}(\mathbb{R})$. By an inequality of Sobolev type,

$$
\|\psi\|_{L_{\infty}}^{2} \leqq \frac{2}{\sqrt{q}} \int_{-\infty}^{\infty} d x\left(\psi^{\prime 2}+q \psi^{2}\right) .
$$

Suppose $\psi$ as in step 5). Choose $\varepsilon>0$ so that $u^{\prime \prime}(a+s)>u^{\prime \prime}(a)-(\beta / 2)\left(1+K_{q}\right)^{2}$ for $a_{-} \leqq a \leqq a_{+}$and $-\varepsilon \leqq s \leqq \varepsilon$. If $\left(\int d x\left(\psi^{\prime 2}+q \psi^{2}\right)\right)^{1 / 2} \leqq \frac{\varepsilon q^{1 / 4}}{\sqrt{2}} \equiv r$, it follows that $\|\psi\|_{L_{\infty}} \leqq \varepsilon$ and

$$
\begin{aligned}
V(\phi+\psi)-V(\phi) & =\int_{-\infty}^{\infty} d x\left\{\frac{1}{2} \psi^{\prime 2}+U(\phi+\psi)-U(\phi)-U^{\prime}(\phi) \psi\right\} \\
& \geqq \int_{-\infty}^{\infty} d x\left\{\frac{1}{2} \psi^{\prime 2}+\frac{1}{2}\left(U^{\prime \prime}(\phi)-\frac{\beta}{2\left(1+K_{q}\right)^{2}}\right) \psi^{2}\right\} \\
& \geqq \frac{1}{2}\left[\left(\psi, V^{\prime \prime}(\phi) \psi\right)-\frac{\beta}{2\left(1+K_{q}\right)^{2}} \int_{-\infty}^{\infty} d x \psi^{2}\right] \\
& \geqq \frac{\beta}{4\left(1+K_{q}\right)^{2}} \int_{-\infty}^{\infty} d x \psi^{2} .
\end{aligned}
$$

Also $U(\phi+\psi)-U(\phi)-U^{\prime}(\phi) \psi \geqq-\frac{1}{2} B_{1} \psi^{2}$, where

$$
\begin{aligned}
& -B=\inf _{x} U^{\prime \prime}(\phi(x)), \\
& B_{1}=B+\frac{\beta}{2\left(1+K_{q}\right)^{2}} .
\end{aligned}
$$

So

$$
\begin{gathered}
V(\phi+\psi)-V(\phi) \geqq \max \left\{\frac{\beta}{4\left(1+K_{q}\right)^{2}}\|\psi\|_{L_{2}}^{2},\right. \\
\left.\frac{1}{2}\left(\int_{-\infty}^{\infty} d x\left(\psi^{\prime 2}+q \psi^{2}\right)-\left(B_{1}+q\right)\|\psi\|_{L_{2}}^{2}\right)\right\} \\
\geqq \frac{\delta}{2} \int_{-\infty}^{\infty} d x\left(\psi^{\prime 2}+q \psi^{2}\right)+\left\{-\frac{1}{2} \delta\left(B_{1}+q\right)+(1-\delta) \frac{\beta}{4\left(1+K_{q}\right)^{2}}\right\} \int_{-\infty}^{\infty} d x \psi^{2}
\end{gathered}
$$

for any $0 \leqq \delta \leqq 1$. Choose $\delta$ such as to make

$$
-\frac{1}{2} \delta\left(B_{1}+q\right)+(1-\delta) \frac{\beta}{4\left(1+K_{q}\right)^{2}}=0 .
$$


so as to get

$$
\begin{aligned}
V(\phi+\psi)-V(\phi) & \geqq K \int_{-\infty}^{\infty} d x\left(\psi^{\prime 2}+q \psi^{2}\right) \\
K & =\frac{\beta / 4}{\beta+\left(1+K_{q}\right)^{2}\left(B_{1}+q\right)} .
\end{aligned}
$$

By (6), (7) and $V(\phi(.+c)=V(\phi)$ we obtain finally

$$
d_{q}(u) \leqq r \Rightarrow V(u) \geqq V(\phi)+K d_{q}(u)^{2} .
$$

In particular, $E(u) \geqq E_{0}+K d_{q}(u)^{2}$.

Proof of Theorem 1. Suppose $d(u(., 0))<r, E=\int_{-\infty}^{\infty} d x\left(\frac{1}{2} u_{t}^{2}\right)+V(u)<V(\phi)+K r^{2}$ at $t=0$, and $u_{t t}-u_{x x}+U^{\prime}(u)=0$ for $t>0$ (the equation is reversible, $t \rightarrow-t$, so we need only consider $t>0$. There exists a unique mild solution with $\left\{u_{x}(., t), u_{t}(., t)\right\} \in$ $L_{2} \times L_{2}$ continuous in $t$ and with constant energy $E$ which exists on some maximal interval $0 \leqq t<t_{\infty}$. If $t_{\infty}<+\infty,\left\{u_{x}(., t), u_{t}(., t)\right\}$ cannot converge in $L_{2} \times L_{2}$ as $t \rightarrow t_{\infty}$ - so $\left\|U^{\prime}(u(., t))\right\|_{L_{2}}$ must be unbounded as $t \rightarrow t_{\infty}$. But on $0 \leqq t<t_{\infty}$, ) $V(\phi)+K r^{2}>E \geqq V(\phi)+K d(u)^{2}$ so $d(u(., t))<r$. Thus for each $t$ there is a $c=c(t)$ so that

$$
\int_{-\infty}^{\infty} d x\left[\left(u_{x}-\phi^{\prime}(x+c)\right)^{2}+q(u-\phi(x+c))^{2}\right]<r^{2} .
$$

So

$$
\left|U^{\prime}(u(x, t))\right| \leqq\left|U^{\prime}(\phi(x+c))\right|+\text { const }|u(x, t)-\phi(x+c)|
$$

for all $x \in \mathbb{R}$, so that $\left\|U^{\prime}(u(., t))\right\|_{L_{2}}$ is uniformly bounded as $t \rightarrow t_{\infty}$. Hence $t_{\infty}=+\infty$ and the solutions exist for all $t \geqq 0$ - and similarly for all $t \leqq 0$.

Remark. If the initial values have $\left\{u_{x x}(., 0), u_{x t}(., 0)\right\} \in L_{2} \times L_{2}$ as well, then $t \rightarrow\left\{u_{x}(., t), u_{t}(., t)\right\} \in L_{2} \times L_{2}$ is continuously differentiable for all $t$ and we have a strict solution (see, for example, [10], Th. VIII, 3.2).

\section{Instability}

The following theorem is featured along the lines of reference [16].

Theorem 2. Let $X$ be a Banach space, $U \in X$ an open set containing 0 , suppose $T: U \rightarrow X$ has $T(0)=0$, and for some $p>1$ and continuous linear $L$ with spectral radius $r(L)>1$.

$$
\|T(x)-L x\|=0\left(\|x\|^{p}\right) \quad \text { as } \quad x \rightarrow 0 .
$$

Then 0 is unstable as a fixed point of $T$.

In fact, we may estimate the direction in which points move away from 0 under successive applications of $T$. Choose any positive integer $m$ and any $\mu, 0<\mu<1 / \sqrt{2}$, 


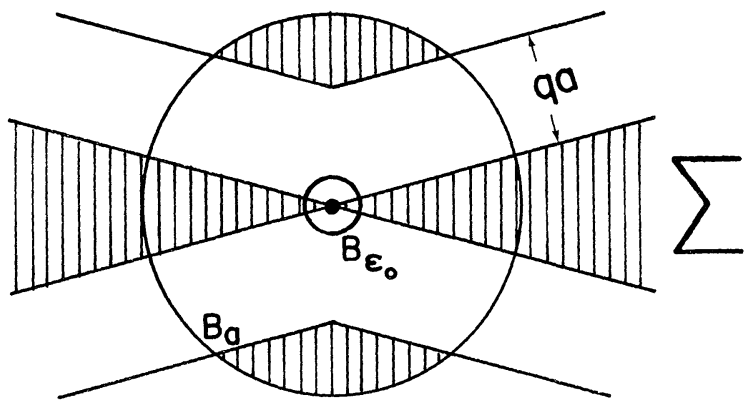

Fig. 1.

and define the cone

$$
\sum=\sum(m, \mu)=\left\{v \in X:\left\|L^{m} v\right\| \leqq \mu r^{m}\|v\|\right\},
$$

where $r=r(L)$. If $0<q<(1 / \sqrt{2}-\mu) /\left(\mu+r^{-m}\left\|L^{m}\right\|\right)$ there exists $a_{q}>0$ such that: given any $a$ in $0<a \leqq a_{q}$, arbitrarily small $\varepsilon_{0}>0$ and arbitrarily large $N_{0}>0$, there exist $N>N_{0}$ and $x \in X$ such that $\|x\| \leqq \varepsilon_{0} .\left\|T^{n}(x)\right\| \leqq a$ for $0 \leqq n \leqq N$ and $\operatorname{dist}\left(T^{N}(x)\right.$, $\Sigma) \geqq q a$. In particular, $\left\|T^{N}(x)\right\| \geqq q a$.

Remark. If $\Gamma$ is a $C^{1}$ curve of fixed points of $T$ with $0 \in \Gamma$, the tangent of $\Gamma$ at 0 will be in $N(L-1) \in \Sigma(M, 1 / 2)$ if $\left.r^{m} \geqq 2\right)$. Choosing $a$ small, so $\Gamma$ is close to its tangent, we may conclude there exist arbitrarily small $x$ such that, for some $N,\left\|T^{n}(x)\right\| \leqq a$ when $0 \leqq n \leqq N$ but $\operatorname{dist}\left(T^{N}(x), \Gamma \cap B_{a}\right) \geqq \frac{1}{2} q a$. (Here $B_{a}$ is the ball of radius $a$ about 0 .) Thus the points $\left\{T^{n}(x), n \geqq 0\right\}$ not only move away from 0 , but also away from $\Gamma$ : the curve $\Gamma$ is unstable.

Proof. Since $r=r(L)>1, p>1$, we may choose $\eta$ in $0<\eta<r^{p}-r$, and then choose $K$ so $\left\|L^{n}\right\| \leqq K(r+\eta)^{n}$ for all $n \geqq 0$. There exist $a_{0}>0$ and $b$, so $\|x\| \leqq a_{0}$ implies $x \in U$ and $\|T(x)-L x\| \leqq b\|x\|^{p}$. Given $\mu, m, q$ as above, choose $\delta>0$ and $0<a_{q} \leqq a_{0}$ so small that

$$
\begin{aligned}
& \Delta \equiv \frac{b K}{r^{p}-r-\eta} a_{q}^{p-1}<1 \quad \text { and } \\
& q \leqq \frac{1-\Delta}{\sqrt{2}+\delta} \cdot C_{\delta}-\Delta, \quad C_{\delta}=(1-3 \delta-\mu(\sqrt{2}+\delta)) /\left(\mu+r^{-M}\left\|L^{M}\right\|\right) .
\end{aligned}
$$

Choose $\lambda \in \sigma(L)$ with $|\lambda|=r$, say $\lambda=r e^{i \theta}$, and choose $N \geqq N_{0}$ so $r^{N} \geqq a_{0} / \varepsilon_{0}$ and $\left|e^{i(N+m) \theta}-1\right|<\delta$. Since $\lambda$ is in the boundary of the spectrum, it is an approximate eigenvalue and there exist $\zeta \in X+i X$ (the complexification of $X$ ) with $\|\zeta\| \geqq 1$ but $\|L \zeta-\lambda \zeta\|$ arbitrarily small. Choose $\zeta=\xi+i \eta(\xi, \eta$ in $X)$ so $\|\xi\|=1 \geqq\|\eta\|$ and for $0 \leqq n \leqq N+m \quad\left\|\operatorname{Re}\left(L^{n} \zeta-\lambda^{n} \zeta\right)\right\|=\left\|L^{n} \xi-r^{n}(\cos n \theta \xi-\sin n \theta \eta)\right\| \leqq \delta r^{n}$. Note $\left\|L^{n} \xi\right\|$ $\leqq(\sqrt{ } 2+\delta) r^{n}$ and $L^{N+m} \xi \| \geqq r^{N+m}(1-3 \delta)$. We prove $\operatorname{dist}\left(L^{N} \xi, \Sigma\right) \geqq C_{\delta} r^{N}$, by contradiction. If $v \in \Sigma$ and $\left\|L^{N} \xi-v\right\|<C_{\delta} r^{N}$, then $\|v\|<\left(C_{\delta}+\sqrt{ } 2+\delta\right) r^{N}$ and $C_{\delta} r^{N}\left\|L^{m}\right\|$ 
$>\left\|L^{m}\right\| \quad\left\|L^{n} \xi-v\right\| \geqq\left\|L^{N+m} \xi-L^{m} v\right\|>r^{N+m}(1-3 \delta)-\mu r^{m+N}\left(C_{\delta}+\sqrt{2}+\delta\right)=$ $C_{\delta} r^{N}\left\|L^{m}\right\|$, a contradiction.

Now let $0<a \leqq a_{q}, R=(\sqrt{ } 2+\delta) /(1-\Delta)$, and $\varepsilon=a / R r^{N}$. Note $a \leqq a_{0}$ and $\varepsilon \leqq a_{0} / r^{N} \leqq \varepsilon_{0}$. Let $x_{0}=\varepsilon \xi, x_{n+1}=T\left(x_{n}\right)$ for $n \geqq 0$; then

$$
X_{n}=L^{n} x_{0}+\sum_{k=0}^{n-1} L^{n-1-k}\left(T\left(x_{k}\right)-L x_{k}\right) \text {. }
$$

Suppose $n \leqq N$ and $\left\|x_{k}\right\| \leqq \varepsilon R r^{k}$ for $0 \leqq k \leqq n-1$; this is certainly true for $n=1$. It follows that

$$
\left\|X_{n}-L^{n} x_{0}\right\| \leqq \sum_{k=0}^{n-1} K(r+\eta)^{n-1-k} b\left(\varepsilon R r^{k}\right)^{p} \leqq \frac{b K\left(\varepsilon R r^{n}\right)^{p}}{r^{p}-r-\eta} \leqq \Delta \varepsilon R r^{n},
$$

so $\left\|x_{n}\right\| \leqq(\sqrt{2}+\delta+\Delta R) \varepsilon r^{n}=\varepsilon R r^{n}$. By induction, $\left\|x_{n}\right\| \leqq \varepsilon R r^{n} \leqq a$ for all $n \leqq N$, and $\left\|x_{N}-L^{N} x_{0}\right\| \leqq \Delta \varepsilon R r^{N}$. Finally dist $\left(L^{N} x_{0}, \Sigma\right)=\varepsilon$ dist $\left(L^{N} \xi, \Sigma\right) \geqq C_{\delta} \varepsilon r^{N}$, so dist $\left(x_{N}, \Sigma\right) \geqq\left(C_{\delta} / R-\Delta\right)_{\varepsilon} R r^{N} \geqq q a$.

\section{Applications}

Solitary-wave solutions of (0-1) are of type

$$
\Phi_{0}(x, t)=\phi_{0}(x-u t)
$$

for suitable $u$. In order to apply Theorems 1 and 2 to the stability of (3-1), we first transform to a fixed-point problem by a Lorentz-transformation (which leaves $(0-1)$ invariant)

$$
x \rightarrow \xi=\frac{x-u t}{\sqrt{1-u^{2}}}, \quad t \rightarrow \tau=\frac{t-u x}{\sqrt{1-u^{2}}} .
$$

(3-1) describes then (for $|u|<1$ ) static (i.e., $\tau$-independent) solutions of $(0-1)$. In one space-dimension, $s=1$, there are two types of nontrivial solutions of the form (3-1) (see Fig. 2):

a) solutions joining two distinct absolute minima $a_{-}$and $a_{+}$of $U: \lim _{x \rightarrow-\infty} \phi(x)$ $=a_{-}<a_{+}=\lim _{x \rightarrow+\infty} \phi(x)$, with $U\left(a_{-}\right)=U\left(a_{+}\right)$; or solutions joining two distinct relative minima $b_{-}<b_{+}$, with $U\left(b_{-}\right)=U\left(b_{+}\right)$;

b) solutions around a relative minimum $\alpha$ of $U: \lim _{x \rightarrow-\infty} \phi(x)=\alpha=\lim _{x \rightarrow+\infty} \phi(x)$. In case a), Theorem 1 applies directly yielding form-stability of the solitary-wave ([4], [6]).

The following condition follows from $b$ ):

c) sgn $\phi^{\prime}(x)$ is not constant in $x \in \mathbb{R}$.

In view of the wealth of solutions for $s>1([13],[17])$, we now state the appropriate analogous conditions for general $s$ :

c1) $\lim \phi(x)=\alpha$ and $\alpha_{1} \equiv U^{\prime \prime}(\alpha)>0$ and $\Phi(\cdot)$ is not constant; $|x| \rightarrow \infty$

c2) $[\phi(\cdot)-\alpha] \in L_{2}\left(\mathbb{R}^{s}\right)$; 


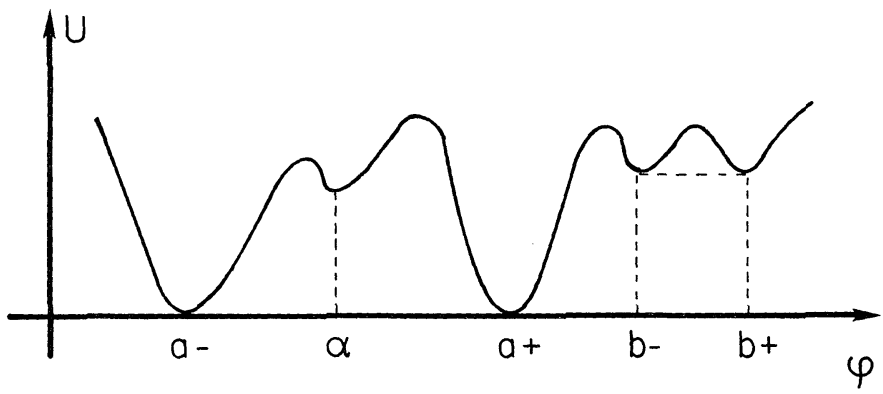

Fig. 2.

In Theorem $2 F_{t}(t \geqq 0)$ is a (nonlinear) semigroup and $T$ is $F_{t}$ at any fixed $t>0$. $L=D F_{t}(0)=\exp (t A)$, where $A$ is the linearized operator

$$
A \equiv\left(\begin{array}{cc}
0 & 1 \\
-K & 0
\end{array}\right)
$$

on $H_{\alpha_{1}}^{1}\left(\mathbb{R}^{s}\right) \oplus L_{2}\left(\mathbb{R}^{s}\right)$, where

$$
\left.H_{\alpha_{1}}^{1}\left(\mathbb{R}^{s}\right)=\left\{u:\|u\|_{\delta_{1}}^{2} \equiv\left(-\Delta+\alpha_{1}\right)^{1 / 2} u,\left(-\Delta+\alpha_{1}\right)^{1 / 2} u\right)<\infty\right\}
$$

and

$$
K \equiv-\Delta+U^{\prime \prime}(\phi)
$$

We have

$$
A=\left(\begin{array}{cc}
0 & 1 \\
-\Delta+\alpha_{1} & 0
\end{array}\right)+\left(\begin{array}{cc}
0 & 0 \\
U^{\prime \prime}(\phi)-\alpha_{1} & 0
\end{array}\right)
$$

The first operator is skew adjoint on $D\left(\left(-\Delta+\alpha_{1}\right)\right) \oplus D\left(\left(-\Delta+\alpha_{1}\right)^{1 / 2}\right)([15]$, Theorem 1, pg. 26). The multiplication operator $\left(U^{\prime \prime}(\phi)-\alpha_{1}\right)$ is by c2) a relatively compact perturbation $\left(-\Delta+\alpha_{1}\right)$ on $L_{2}\left(\mathbb{R}^{s}\right)$, hence it is a compact operator on $H_{\alpha_{1}}^{\prime}\left(\mathbb{R}^{s}\right)$, and $\left(\begin{array}{cc}0 & 0 \\ U^{\prime \prime}(\phi)-\alpha_{1} & 0\end{array}\right)$ is a compact operator on $H_{\alpha_{1}}^{1}\left(\mathbb{R}^{s}\right) \oplus L_{2}\left(\mathbb{R}^{s}\right)$. Therefore $|\operatorname{Re} \sigma(A)| \leqq$ constant, and $L=\exp (t A)$ exists as a semigroup of bounded linear operators. We have now

Proposition 2. Under assumption (c) $($ for $s=1)$ or $\mathrm{c} 1)$ and $\mathrm{c} 2)($ for $s>1), K$ has an eigenvalue $e_{0}<0$ and $A$ has an eigenvalue $\sqrt{\left|e_{0}\right|}>0$, so $r(L)>1$.

Proof. It follows from c1) and c2) that $\left(U^{\prime \prime}(\phi)-\alpha_{1}\right)$ is a relatively compact perturbation of $\left(-\Delta+\alpha_{1}\right)$, as remarked above. Hence $\sigma(K) \backslash\left[\alpha_{1}, \infty\right]$ consists of point eigenvalues of finite multiplicities which can accumulate at most at $\alpha_{1}$. We have

$$
K \partial_{x_{i}} \phi=0, \quad i=1, \ldots, s .
$$

Hence (by (c3)) zero is a discrete eigenvalue, and the bottom of $\sigma(K)$ is an eigenvalue $e_{0}$. By ([12], theorems XIII-43 and XIII-45), $K$ is ergodic and by ([12], theorem 
XIII-43) $e_{0}$ is a simple eigenvalue and the corresponding eigenfunction $\psi \in D(K)=$ $D(\Delta)$ is strictly positive. But by c) (for $s=1) \partial_{x} \phi \neq \lambda \Psi$. For $s>1, \partial x_{i} \phi \neq \lambda \Psi$ for all $i=1, \ldots, s$ because $\Psi$ is simple and $\partial_{x_{i}} \phi \neq \lambda \partial_{x_{j}} \phi$ for some $i \neq j$ (i.e., the zero eigenvalue is degenerate). To see this, suppose $\sum_{1}^{s} \lambda_{j} \partial_{x_{j}} B \equiv 0$ for some constant $\lambda \in \mathbb{R}^{s}$. This implies

$$
\frac{d}{d t} \phi(x+\lambda t)=0, \quad \phi(x)=\phi(x+\lambda t)
$$

for all $x, t:$ if $\lambda \neq 0$, let $t \rightarrow \infty$ and conclude from $c 1$ ) that $\phi(x)=\alpha$ for all $x$. Hence, in all cases, $e_{0}<0$. Let $\beta=-\left|e_{0}\right|$. The vector

$$
v=\left(\begin{array}{cc}
\psi & \\
\sqrt{\beta} & \psi
\end{array}\right) \in L_{2}\left(\mathbb{R}^{s}\right) \oplus L_{2}\left(\mathbb{R}^{s}\right)
$$

is an eigenvector of $A$ corresponding to eigenvalue $\sqrt{\beta}>0$. But $\psi \in D(\Delta)$, hence $\psi \in H_{\alpha_{1}}^{1}\left(\mathbb{R}^{s}\right)$ and $v \in H_{\alpha_{1}}^{1}\left(\mathbb{R}^{s}\right) \oplus L_{2}\left(\mathbb{R}^{s}\right)$.

The above proposition proves instability for case b) (and $s=1$ ) under assumption (3). There are many examples in higher dimensions ([13], [17]). For an explicit example in dimension $s=3$, consider the following potential ([13]):

$$
U(\phi)= \begin{cases}g\left[(\phi-c)^{3}-3(\phi-c)^{4}\right], & c \leqq \phi \leqq 1+c, \\ g(\phi-c)^{4}, & \text { for } \phi \leqq c, \\ f(\phi), & \text { for } \phi \geqq 1+c,\end{cases}
$$

where $f \in C^{2}$, smoothly matched at $\phi=1+c$, with $\left|f^{\prime \prime}(\phi)\right| \leqq$ const $\left(1+\phi^{2}\right), g>0$ and $c$ is a given constant. $U \in C^{2}$, and $\phi=c$ is a relative minimum. The function

$$
\phi(r)=c+\left[1+\left(\frac{r}{a}\right)^{2}\right]^{-1}
$$

with $a^{2}=2 / 3 g$, is a radial solution of $(0-1)$. It satisfies $\left.\mathrm{c} 1\right)$ and $\left.\mathrm{c} 2\right)$. Proposition 2 , coupled with Theorem 2, implies therefore that the above solution is unstable, as conjectured in [13]. Similarly, the (infinite series of) radial solitary-wave solutions of $(0-1)$ constructed by Strauss in [17] are unstable, by the same reasoning.

The above results lead us to revisit the Derrick-Strauss theorem ([7], [8]). The latter states (in the form originally proposed in [7]) that solitary-wave solutions of scalar field theories do not exist for $s>1$, provided they have finite energy relative to the absolute minimum (or minima) of $U$. However, solutions joining two relative minima of $U$ (as $b_{-}$and $b_{+}$in Fig. 1) are stable and define a Hilbert sector, although they have infinite energy relative to the absolute minima. Hence, the finite energy property does not seem to be relevant. If we accept this, there exist solutions around relative minima of $U$ (as defined in b)) both for $s=1$ and $s>1$, as the previous examples show. The latter are, however, unstable. Stability seems therefore to be the main issue. 


\section{References}

1. Lax, P. D.: Comm. Pure Appl. Math. 21, 467 (1968)

2. Coleman, S.: Classical lumps and their quantum descendants. Lectures at the 1975 International School of Subnuclear Physics "Ettore Majorana" (Erice), in "New Phenomena in Subnuclear Physics.” Zichichi A. (ed.). New York: Plenum Press, 1977

3. Hepp, K.: Comm. Math. Phys. 35, 265 (1974)

4. Benjamin, T. B.: Proc. R. Soc. (London) A328, 153 (1972)

5. Bona, J. L.: Proc. R. Soc. (London) A344, 363 (1975)

6. Scharf, G., Wreszinski, W. F.: Ann. Phys. (N.Y.) 134, 56 (1981)

7. Derrick, G.: J. Math. Phys. 5, 1252 (1964)

8. Strauss, W.: Nonlinear invariant wave equations. In: Invariant Wave Equations, Velo, G., Wightman, A. S. (eds.), Berlin, Heidelberg, New York: Springer 1977

9. Dunford, N., Schwartz, J.: Linear operators, Vol. II. New York: Interscience 1963

10 Martin, R. H.: Nonlinear operators and differential equations in Banach spaces. New York: Wiley 1976

11. Reed, M., Simon, B.: Methods of modern mathematical physics, Vol. IV. New York: Academic Press 1978

12. Parenti, C., Strocchi, F., Velo, G.: In: Invariant Wave Equations, Velo, G., Wightman, A. S.: (eds.), Berlin, Heidelberg, New York: Springer 1977, and Commun. Math. Phys. 53, 65 (1977)

13. Parenti, C., Strocchi, F., Velo, G.: Dynamical charges and symmetries in non-linear classical field theory. Bielefeld Preprint

14. Chernoff, P. R., Marsden, J. E.: properties of infinite dimensional Hamiltonian systems. Berlin, Heidelberg, New York: Springer 1974

15. Marsden, J., McCracken, M.: The Hopf bifurcation and its applications. Berlin, Heidelberg, New York: Springer 1976

16. Henry, Daniel B.: Stability and instability by the linear approximation, Proc. of the 12th. Brazilian Analysis Seminar (Soc. Bras. de Matematica, Rio de Janeiro)

17. Strauss, W. A.: Commun. Math. Phys. 55, 149 (1977)

Communicated by J. Moser

Received August 28, 1981 
